\title{
REAL-TIME MONITORING SYSTEM USING UNMANNED AERIAL VEHICLE INTEGRATED WITH SENSOR OBSERVATION SERVICE
}

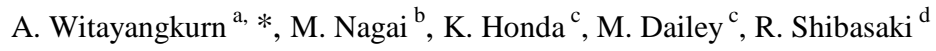 \\ ${ }^{a}$ Dept. of Civil Engineering, The University of Tokyo, Institute of Industrial Science Cw-503 4-6-1 Komaba Tokyo, Japan - \\ apichon@iis.u-tokyo.ac.jp \\ ${ }^{\mathrm{b}}$ Geoinformatics Center, Asian Institute of Technology, P.O.Box 4 Klong Luang Pathumtani, Thailand - nagaim@ait.ac.th \\ ${ }^{\mathrm{c}}$ School of Engineering and Technology, Asian Institute of Technology, P.O.Box 4 Klong Luang Pathumtani, Thailand - \\ honda@ait.ac.th, mdailey@ait.ac.th \\ ${ }^{d}$ Center for Spatial Information Science, The University of Tokyo, 5-1-5 Kashiwanoha Kashiwa-shi Chiba, Japan - \\ shiba@csis.u-tokyo.ac.jp
}

Commission I, WG I/V

KEY WORDS: UAVs, Real-time monitoring, Sensor network, Sensor Service Grid, Sensor Observation Service

\begin{abstract}
:
The Unmanned Aerial Vehicle (UAV) is an emerging technology being adapted for a wide range of applications. Real-time monitoring is essential to enhance the effectiveness of UAV applications. Sensor networks are networks constructed from various sensor nodes. International standard such as OGC's SOS (Sensor Observation Service) makes it possible to share sensor data with other systems as well as to provide accessibility to globally distributed users. In this paper, we propose a system combining UAV technology and sensor network technology to use an UAV as a mobile node of sensor network so that the sensor data from UAV is published and shared real-time. A UAV can extend the observation range of a sensor network to remote areas where it is usually difficult to access such as disaster area. We constructed a UAV system using remote-controlled helicopter and various sensors such as GPS, gyrocompass, laser range finder, Digital camera and Thermometer. Furthermore, we extended the Sensor Observation Service (SOS) and Sensor Service Grid (SSG) to support mobile sensor nodes. Then, we conducted experiments of flying the helicopter over an area of the interest. During the flight, the system measured environmental data using its sensors and captured images of the ground. The data was sent to a SOS node as the ground station via Wi-Fi which was published using SSG to give realtime access to globally distributed users.
\end{abstract}

\section{INTRODUCTION}

\subsection{Background}

Many countries have high risk of natural disasters such as earthquakes, typhoons, and volcanic eruptions. Disaster areas are very dangerous, especially in recent cases. Roads, bridges and other transportation services are usually interrupted. Therefore, it is difficult to recover areas and also danger for teams which are sent to rescue people trapped in the area. Not only are recoveries and rescue process concerned, but prevention procedures are also in focus. Since some areas are often faced with disasters, it would be better for people in those areas to know when it will happen or at least to forecast the situation. This could reduce loss and damage because people would have time to plan and prepare for the situation by evacuating the area. However, prevention requires a lot of data and information from many types of sensors such as rain sensors, soil sensors and temperature sensors. To collect the data, sensor stations can be setup in particular areas sending data back to a central station. Unfortunately, in some places, settings up stations are difficult, especially in very remote areas. Electricity and communication services are usually serious concerned. Moreover, to reach the highest efficiency for both proactive and corrective process, sensor data must be consolidated and analysed from many sources. There are many research centers nowadays, but there is a little sharing of data because there is no actual system that is widely used, even though a standard called Observations and Measurements Encoding Standard (O\&M). O\&M only describes an abstract model and XML Schema for sensor observations as well as a general framework, so it is still difficult to implement a system from the standard.

The Unmanned Aerial Vehicle (UAV) is an emerging technology being adapted for use in a wide range of applications such as remote sensing, scientific research, and search and rescue since it is a pilotless airplane controlled by radio remote control autonomous flight. Hence, it is suitable for UAVs to operate in disaster areas for monitoring, observing, and gathering needed information for rescue teams. This is safer and faster than sending teams to the area. Once the UAV operation has acquired the necessary information and analysed the data, teams can be deployed more efficiently. Furthermore, collected information can be sent to central agencies. They can access information from anywhere and take action within a short period of time.

\subsection{Motivation}

In this work, a system was built on an existing sensor network named SensorAsia. It consists of 2 main services: Sensor

\footnotetext{
* Corresponding author. This is useful to know for communication with the appropriate person in cases with more than one author.
} 
Observation Service (SOS) and Sensor Service Grid (SSG). The Sensor Observation Service runs on sensor stations that handle data collection in the field. The Sensor Service Grid is a system that can be accessed from the Internet. It provides sensor data synchronized from SOS station and grants access to SOS stations, allowing remote configuration as well as monitoring stations from the Internet. However, SOS and SSG were designed and developed based on a fixed-position sensor station assumption, which is not flexible enough for some situations such as ad-hoc nodes and mobile stations. For example, in a disaster area, it may not be possible to install a new sensor station; stations already installed might be affected by the disaster. The UAV systems held in a hot research field because it can be adapted for use in various applications and also address limitations in other research areas. It can fly over an area with no transportation service required. Real-time infrastructure surveillance, forest fire monitoring, traffic monitoring and disaster monitoring are some example applications of UAV systems. Individual UAVs are usually instrumented with sensors and geosensors such as GPS, gyrocompass and digital cameras. They operate as standalone systems or are limited to a group of UAVs with a proprietary architecture, meaning that even though it can work properly, it may not be able to communicate and share sensor data with other UAVs or sensor networks. Therefore, by extending SOS and SSG to unmanned aerial vehicle based sensor station, we got dual benefits. Sensor Asia provides standardization, and the UAV based sensor station extends Sensor Asia's observation range to difficult areas. The idea for this research is that a UAV loaded with sensors and cameras to fly by remote control to a disaster area to collect sensor data such as temperature and humidity while capturing images on its flight. It transmits data to ground station units and the Sensor Service Grid for accessing from the Internet and further analysis.

\subsection{Goal and Objectives}

The main goal of this study is to design and develop a UAV system as a node of a field sensor network based on SOS and SSG, aiming to achieve the following tasks: 1 . Design and implement a UAV system for real-time monitoring including framework, architecture and components. 2. Connect sensors and geosensors such as GPS, gyrocompass, digital camera and laser ranger to a main controller for acquiring, processing and sending data to a ground station unit. 3. Extend the UAV System to communicate with SOS and SSG so that it can act as one sensor node. 4. Modify the Sensor Observation Service Station (SOS Station) and Sensor Service Grid (SSG) to support mobile stations. 5. Test and evaluate the system in a flight experiment.

\section{LITERATURE AND RELATED WORK}

\subsection{Sensor Network}

Sensor Network is a group or system of sensors which connect together using communication infrastructure to exchange information. It consists of multiple sensor stations which can be called sensor nodes. All sensor nodes are able to link or synchronize data among each other or main station so that it acts as network. It is deployed in the area that wants to monitor and collect data such as weather and environment. Rural areas or disaster sites are usually installed the station. In this research, we used a sensor network application named SensorAsia. It is a new approach to implement real time sensor network with high density sensor nodes using Fieldserver in Asia in order to acquire various environmental information. The initiative is developing GIS and Sensor integration system called Sensor Service Grid (SSG) to realize easy deployment and uncostly for installation and operation of Fieldserver networks. Under this initiative, field-side agent boxes based on Sensor Observation Service (SOS), called SOS Stations, have been developed. The use of SOS Stations with facilities of the Sensor Service Grid (SSG), under Sensor Asia initiative, can simplify these tasks for general users (Honda et al., 2008). For this research, UAV has been designed to be a sensor node of SOS station and it can be called Aerial Fieldserver (Aerial FS).

\subsection{Unmanned Aerial Vehicle}

An Unmanned Aerial Vehicle (UAV) is a pilotless airplane that can be controlled by radio signal remote or fly autonomously based on flight path programmed or more complicated autonomous systems. UAV can carry many sensors such as gps, compass, laser scanner and digital camera. With those sensors, it can use in various mission in different field such as pollution monitoring, Forest Fire detection and disaster monitoring. Ollero and Alcazar (2003) presented a helicopter teleoperation system for using in forest fire detection and monitoring. It monitors and computes the distances from the fire to houses, road and utilities. Their system consisted of camera and sensors to get helicopter position and environment data. The survey and ground mapping are able to be done by UAV integrated with multi-sensors (Nagai et al., 2009). In our research, we aim to extend and publish local observation to internet and able to observe by far-end concerned person.

\section{SYSTEM OVERVIEW}

The system is designed to support real-time monitoring operation which can collect sensor data and image data by using UAV with payload sensor platform. Data must be viewable by operator at ground station unit as well as central agencies which can access from Internet through Sensor Service Grid (SSG). The overview and flow of the system is shown in Figure 1.

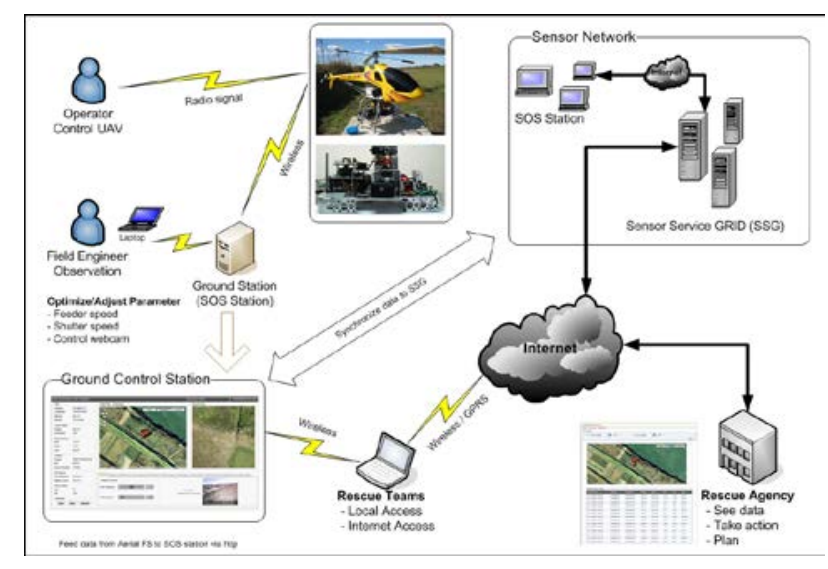

Figure 1. System Overview

\subsection{System Design}

The main system was developed using Java Programming that allows the system to work in any platform such as windows and Linux. However, we used Windows as an operating platform due to limitation in proprietary driver of gyrocompass. Java framework such as Hibernate and Spring were chosen to use in this system to make it standardized, easy to develop and more 
robust. JSP, Servlet and JSTL were used for web presentation. Google Map was used for showing live map and AJAX technology was enhanced to enable more real-time web interface. The figure 2 shows system diagram, architecture and technology related to this research. The system is separated into two parts: UAV System (Aerial FS) and Mobile SOS Station.

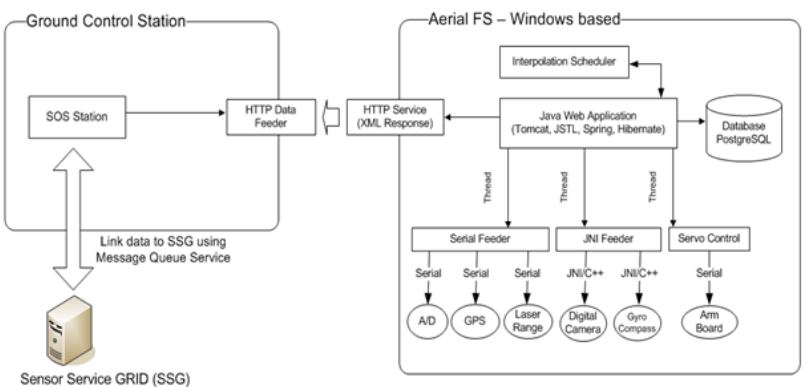

Figure 2. System Diagram and Architecture

For UAV System (Aerial FS), it was developed using Java technology for core system and $\mathrm{C}++$ language for device connecting part. PostgreSQL was chosen to be database because it is a light-weighted, scalable and supporting many platforms. Hibernate and Spring frameworks, which are famous in database management and transaction control, are used as data access layer in this system. Besides, Java Native Interface (JNI) technology is used for connecting native device which has to connect via its proprietary driver. It enables Java to call native library such as Dynamic-Link Library (DLL) in Windows platform and share library in Linux platform. Basically, the system will work as multi-thread; all feeders have their own threads because each device has different data rate such as 1 record per second for GPS while gyrocompass is 180 records per second. This technique allows the system to feed data as maximum speed of each device because it does not need to wait for other devices. Conversely, it generates asynchronous data so that it needs additional process to synchronize all data with time basis. In case of time mismatching or not exactly matching in millisecond level, a basic interpolation was applied to eliminate that problem as well as to get better results. Since this research was focusing on building a real-time system, the only linear interpolation algorithm was applied in an interpolation process. For mobile SOS Station, mobile feature was added to the existing software since SOS station was initially designed on fixed position node and it cannot apply for using with the sensor node which always changes its position such as helicopter. When it flies, position of the sensor is changed according the helicopter position. The position of the sensor can get from INS/GPS sensor which consisted of roll, pitch, yaw, latitude, longitude and velocity. From this concept, position of Fieldservers and sensors can be tied with physical sensors. For example, latitude position of Fieldserver is bound with latitude value of GPS sensor. Each sensor data must also keep the location of itself. Furthermore, we developed a data feeder for feeding data from UAV system. UAV system provides live interpolated data via XML data format as shown in figure 3 and can feed via HTTP. Data included both sensor data and image data. By doing this, it allows users to observe and maintain their data via central servers. Internet connection required for data synchronization; however, it does not have to have a fixed public IP and can be any type of Internet connection such as ADSL, Satellite and GPRS since Message Queue was taken into account under development of SSG.

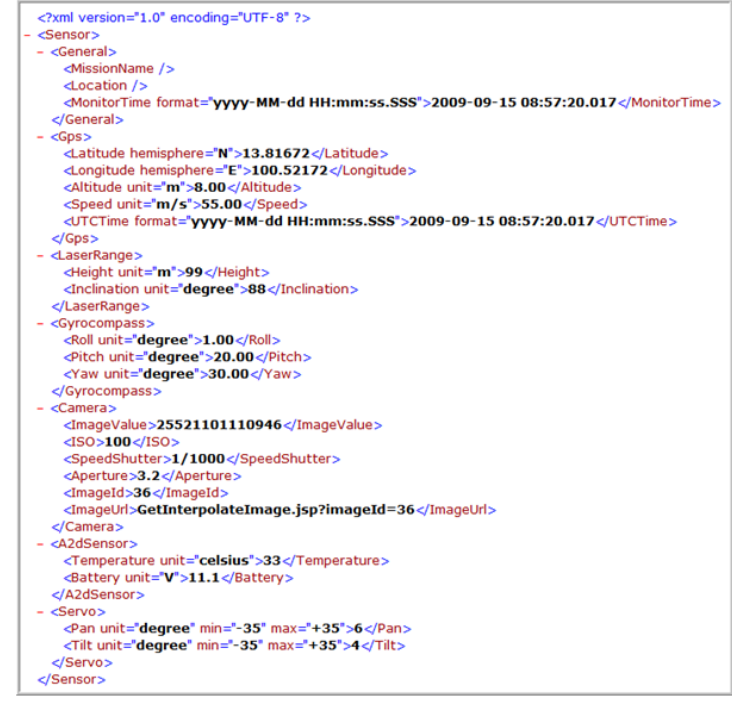

Figure 3. XML Response Format

\subsection{Hardware Platform}

To design platform for UAV, there are several points of concerns. One of the most important points is layout and position of all devices. It needs to be properly aligned, so center of gravity will stay in the center of the plane. A good layout and position design also makes it easy to connect power lines, and internal battery compartment. Besides, the layout should be such that all data and power lines can be connected easily, easy access to battery so that it can be changed easily. Another critical point is a total weight of platform including all equipments. In this experiment, the helicopter has payload approximately 3-4 KG. So, weight of each device has to be concerned for hardware selection. Lastly, since a battery will be used in the platform and it cannot carry a heavy size, power consumption of each equipment has to be minimized (Cai et al., 2008).

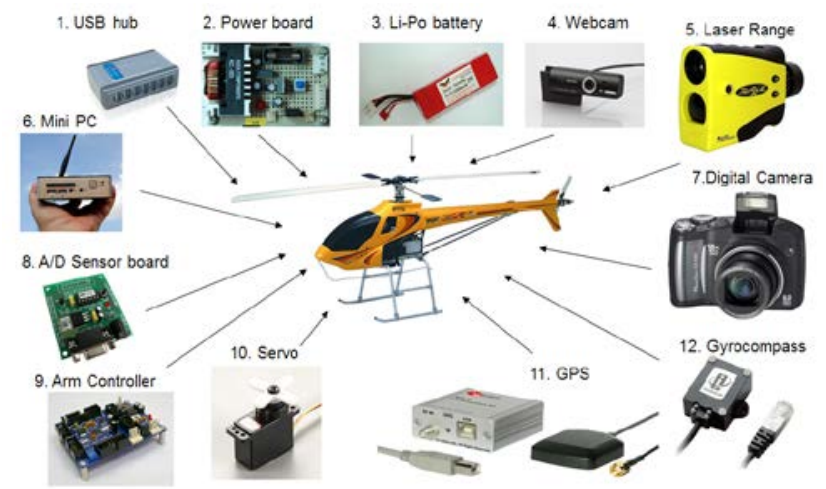

Figure 4. Hardware Equipment

As shown in figure 4, There are many equipments attached to helicopter such as mini PC that act as main controller to feed data from all sensors as well as to provide web access to ground station unit. Digital camera, laser range, gyrocompass and GPS are main sensors for observation. ARM controller board is used for controlling servo to move Webcam position. Li-Po battery can supply power for one hour. For connecting equipment to Mini PC, several types of the connection interface are selected based on device specifications and available ports in Mini PC. 
One additional Usb-Hub has been added for connecting devices since there is not enough port in Mini PC. Gyrocompass and Laser range meter are connected directly to the system using serial interface (RS232) due to performance issue. USB ports are used for connecting a digital camera and a webcam to the PC. GPS, ARM controller board and A/D controller board also used USB interface but they needed an additional converter, USB-to-Serial for connecting because they were native serial devices. All devices that used converter will be mapped as virtual com port in the system. Wireless card was added inside Mini PC to allow wireless connection. In our experiment, when we connected gyrocompass with USB-to-Serial interface, it consumed a lot of CPU time; however, when we connected it using native serial port, it worked fine and did not consume much of CPU time. For Digital camera and Webcam, they were connected directly to mini PC to reduce latency in data transferring between system and Usb-Hub. Also they would not interrupt other devices when transferring huge data. In figure 5 and 6 , it shows layout of the equipment on the platform. The Platform was made from aluminium that had less weight than metal. It was also drilled to make many holds to make it more light-weighted and to have better air flow (Nagai et al., 2009). Gyrocompass was put in the direction pointed to front side of helicopter to make it possible to know directions of the helicopter when flying and it was also orthogonal to the platform. Webcam was put in same direction with gyrocompass to get a clear front view. It was attached on pan-tilt stand that can be controlled via software. Laser range meter and camera are put in the same line and point down to the ground view so that we knew the distance between camera position and the ground. Wireless antenna was put in the right side of the platform. It was a removable antenna so it would be switchable to a bigger one for long range observation. With the $2200 \mathrm{~mA}$ Li-Po battery, system can run up-to one hour. All equipments were using the main battery except the camera and the laser range meter that used their internal AA battery.

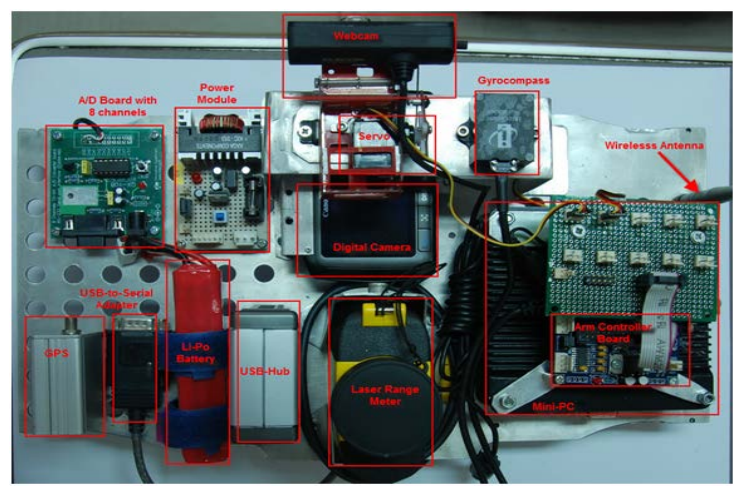

Figure 5. Top-view of Hardware Platform

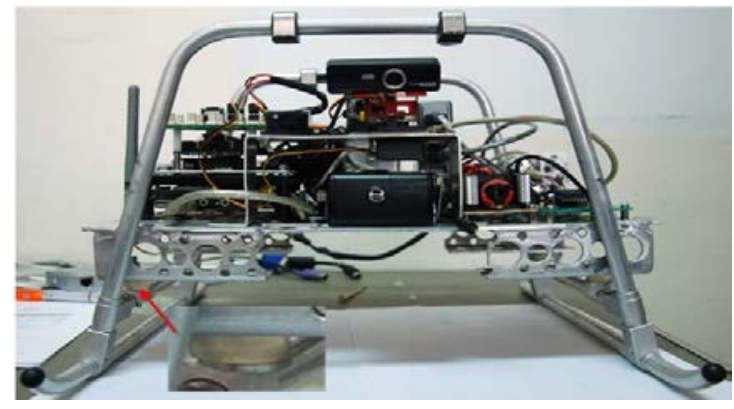

Figure 6. Helicopter attachment and hardware platform

\section{EXPERIMENTAL RESULTS}

The experiment was conducted to test the system. The attachment was tested by hand-held and move around to check the result and reliability at first stage. After that, the hardware platform was attached the helicopter and the flying experiment was tested with full operation. A gasoline engine with $4 \mathrm{~kg}$ payload helicopter, JR Voyager GSR 260, was selected for the experiment. The expected result was that the helicopter could fly without any problems and the system can also run with all sensors working even when flying. The ground station could see the data and send control command via web interface through Wi-Fi. The mobile SOS station at ground station was able to feed data from UAV system as well as to synchronize data to SSG Server in remote location using mobile 3G internet connection within almost real-time condition. The figure 7 shows our experiment setup including all related devices for this experiment. Two batteries were prepared to supply wireless access point and laptop. Laptop, Core2Duo 2.2GHz 4GB Ram, was used as a ground station unit (Mobile SOS Station). It connected to the access point to join the same network as UAV system. To enable internet access, b-mobile $3 G$ modem was used by connecting via USB interface as seen in small picture.

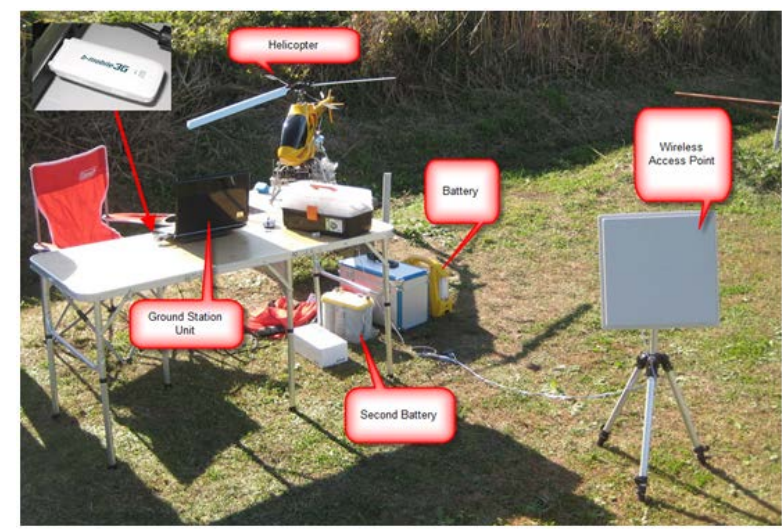

Figure 7. Prepared Equipment for Testing

For flying test, a several flight tests have been done. The figure 8 shows the helicopter with an attachment flying over field area.

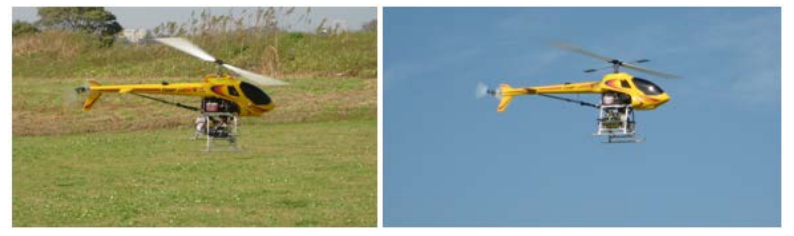

Figure 8. Flight Test on the experiment day

Ground station unit can access to web interface of UAV station using wireless connection. The current data are shown in user interface as shown in figure 9. The user interface consists of five sections: Sensor Data, Flight Path, Ground View, Command and Configuration. Since Ajax technique was used in the system, it did not require users to refresh the webpage to get new data like common webpage. Sensor Data, Point in Google map, Ground view image and webcam image were automatically reloaded based on refreshing time in the configuration. To start and stop data feed; users could simply click start and stop buttons provided on the webpage. 


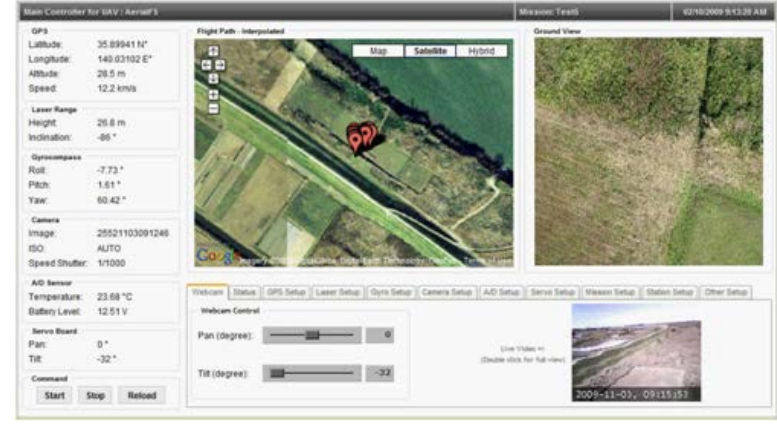

Figure 9. UAV Web Interface during Flight Test

When clicking a point in Google, the system showed a tooltip dialog with three tabs of sensor data. Slidebar Webcam Control interface was provided to control pan and tilt servo. Live webcam video was also displayed in the right part. In the figure 10, it is a user interface of SSG system which show position data synchronized from mobile station. However, only interpolated data will be synchronized to SSG and as for data interpolation, we use image capture time as baseline for calculation.

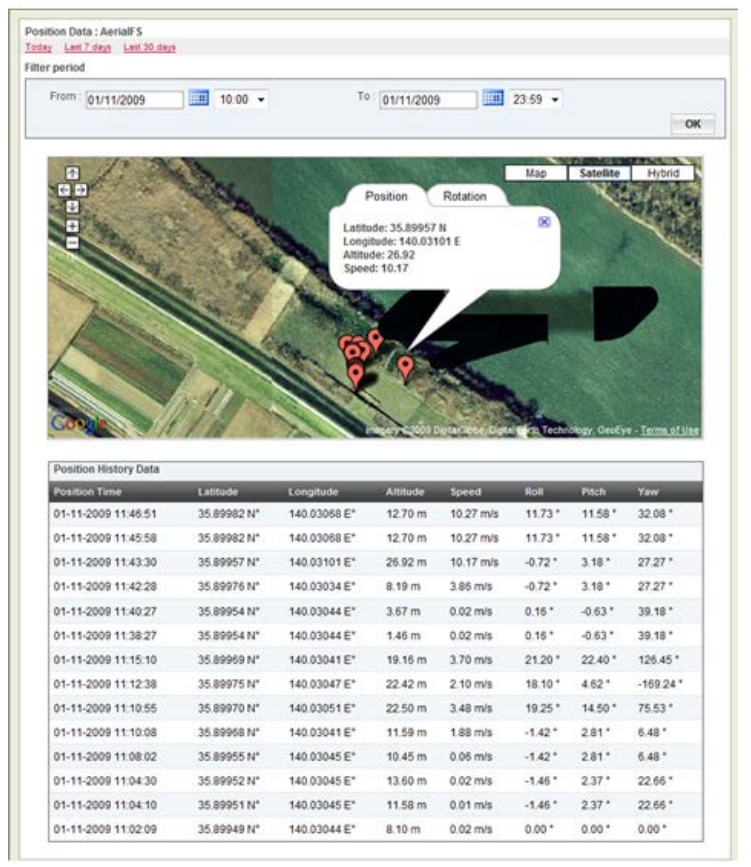

Figure 10. Interpolated data in Sensor Service GRID

By adjusting sensor parameters, the system could run faster and more stable. It could reach one data per second for GPS, Laser Range, Gyrocompass and A/D board. However, camera still could not take more images. As we checked, performance of data rate of each sensor would be decreasing while the camera is capturing images and transferring images to the system. Since we used CF card as data storage for mini pc and it was quite slow compared to standard PC storage. The example of captured images is shown in figure 11. Camera was set to use Shutter Speed Mode at 1/800 and ISO AUTO. Furthermore, the digital camera could take images approximately 20 seconds/image at ground or low altitude; however, when it flew at a higher altitude, taken time became 50-60 seconds/image. We suspected that it might have come from auto-focus function of the camera because the higher it flied, the smaller the objects became on the ground. Moreover, the air vibration also affects the camera. The auto focus used more time to focus the objects before releasing the shutter.

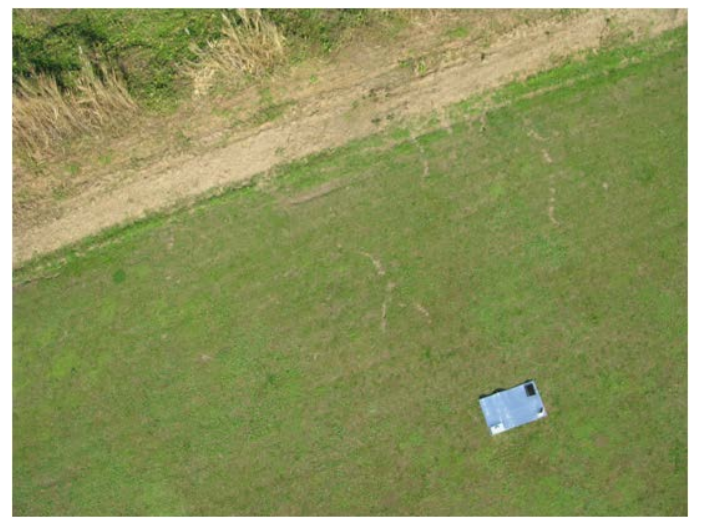

Figure 11. Image from Camera, 1/800, ISO80, F2.8

The overall testing results are shown in table 1. For the camera after the experiment, we have modified data feeder again to make it run on Manual mode instead of a Shutter Speed mode. In manual mode, we were allowed to set the shutter speed, and aperture as well as ISO. Unfortunately, we did not have a chance to do flight test again so as we tested in our laboratory. On a laptop, it could capture one image by 3 seconds and on mini PC, it uses 10 seconds per image which was much better than before.

\begin{tabular}{|c|c|c|c|}
\hline Sensor & $\begin{array}{c}\text { Maximum } \\
\text { Data Rate } \\
\text { (data/sec) }\end{array}$ & $\begin{array}{c}\text { Interval Setting } \\
\text { (second) }\end{array}$ & $\begin{array}{c}\text { Actual Result } \\
\text { (Second) }\end{array}$ \\
\hline Camera & $1 / 3$ & 10 & $\begin{array}{c}\text { 15-20 on ground } \\
50-60 \text { on high altitude }\end{array}$ \\
\hline Gyrocompass & 180 & 0.5 & 0.5 \\
\hline Laser Range & 1 & 1 & 1 \\
\hline GPS & 1 & 1 & 1 \\
\hline A/D & 1 & 1 & 1 \\
\hline
\end{tabular}

Table 1. System Performance of flight experiment0

\section{CONCLUSIONS}

The main objective of this research is to design and develop UAV system as a node of field sensor network which is based on Sensor Observation Service Station and Sensor Service GRID. The development was started from selecting suitable hardware and sensors by using size, weight and power consumption as main criteria because the test-bed helicopter can have only 3-4 kg payloads and battery pack will be used from supplying electricity to all devices. Platform layout is designed and made to fit with all selected devices. Aluminium is used as material for the platform. The sensor devices consist of GPS, Gyrocompass, Laser Range Meter, Digital Camera, Web cam and A/D sensor board. The system is a web-based application developed by Java Programming and use PostgreSQL as database server. Data feeders for each sensor are implemented to connect sensor and feed data. Threads were used to make data feeders run independently. AJAX technology and Google map were used for making interactive user interface. Linear Interpolation process was applied to synchronize data from each sensor by using captured time of image data as base line. HTTP Service for handling requests from the ground station and 
response XML data. On the other side, SensorAsia, a modified sensor work that previously only supported fixed station, is modified to support mobile station and it allowed UAV system to act as a sensor node. It will then can send its data to central server and publish to other people accessible from Internet. As a result of our experiments, total weight of the attachment including the platform and devices is $3.5 \mathrm{~kg}$. The system can run up to one hour with Li-Po battery. Ground station unit is able to access web interface of UAV system to observe live data and status using wireless network deployed in the field. Changing setting of each sensor and controlling sensor can be done as well. We finally could make the system feed data at one record per second for all sensors except digital camera images that are fed every ten seconds. Mobile SOS Station that runs on the ground station unit could obtain interpolated data from UAV system via wireless provided by ground access point (IEEE802.11g, $54 \mathrm{Mbit} / \mathrm{s}$ ) and synchronized data both images and sensors to central node (SSG Server) located in somewhere else using mobile $3 G$ service within a few minutes depending on the 3G internet speed.

\subsection{Limitation}

As concluded, the system has to feed data from many sensors. All feeders are run as threads meaning that sensors are connected and obtained at almost the same time. The current hardware specification of mini PC both CPU and Harddisk could not make it runs in very real-time. Besides, digital camera did not fast enough comparing with usual speed of helicopter to capture images since the limitation of its specification stated that it could take only one image per three second and software development kits from Canon that cannot control some useful function such as turn off auto focus. Webcam can be also treated as limitation because it is connected to the system and need a lot of CPU time to encode live video; hence, it affect the performance of other feeders. According to limitation on controlling the system and power supply for equipments, the operation requirement limit to: the distance is $200-300$ meters; Height is $50-60$ meters; Helicopter speed is $5 \mathrm{~m} / \mathrm{s}$; operation time is 15 minutes.

\subsection{Future Work}

This UAV system can be improved by increasing performance of hardware such as mini pc and digital camera. It will eliminate some of limitation and enable the system to be more real-time. Moreover, Additional sensors can be added to monitor environment such as humidity and CO2 sensor. Ground View Image Stitching is also one of features that can adapt by using data from UAV system as well as mapping image data to Digital Elevation Model (DEM) map. Finally, complex algorithm and model such as Kalman Filter and Particle Filter can be applied for interpolation process to make it more accurate. Furthermore, the exact amount of transmitted data volume and bandwidth of the used Wi-Fi connection must be measured to archive realtime system.

\section{REFERENCES}

Cai, G., Feng, L., Chen, B., Lee, T., 2008. Systematic design methodology and construction of UAV helicopters. Mechatronics, 18(10), pp.545-558.

Honda, K., Chinnachodteeranun, R., Shrestha, A., Witayangkurn, A., Duy, H. N., Shimamura, H., 2008. Field sensor network for environmental monitoring - sensor asia initiative and applications, in: International Symposium on Geoinformatics for Spatial Infrastructure Development in Earth and Allied Sciences, Hanoi, Vietnam.

Honda, K., Shrestha, A., Witayangkurn, A., Chinnachodteeranun, R., Shimamura, H., 2009. Fieldservers and sensors service grid as real-time monitoring infrastructure for ubiquitous sensor networks. Sensors, pp.1-8.

Laser Technology, I., 2005. Lti trupulse $200 / 200 \mathrm{~b}$ user manual. Laser Technology, Inc., CO, USA.

Lawrence, C. T., 2000. Unmanned air vehicles (uavs) for cooperative monitoring (Tech. Rep. No. SAND20000185). Albuquerque, Sandia National Laboratories, CA, USA.

Nagai, M., Tianen, C., Shibasaki, R., Kumagai, H., Ahmed, A., 2009. Uav-borne 3-d mapping system by multisensor integration. IEEE Transactions on Geoscience and Remote Sensing, 47 (3), pp.2371-2388.

Ollero, A., Alcazar, J., Cuesta, F., Nogales, C., 2003. Helicopter Teleoperation for Aerial Monitoring in the COMETS MultiUAV System, in: 3rd Iarp Workshop on Service, Assistive and Personal.

\section{ACKNOWLEDGMENTS}

The authors would like to acknowledge the work of Aadit Shrestha and Rassarin Chinnachodteeranun on helping on platform development and support during all experiments. This research was research collaboration between Asian Institute of Technology and The University of Tokyo; and, it was based on Master Thesis of Apichon Witayangkurn. 